\title{
Field evaluation of 2 collar-mounted activity meters for detecting cows in estrus on a large pasture-grazed dairy farm
}

\author{
C. Kamphuis, ${ }^{1}$ B. DelaRue, C. R. Burke, and J. Jago \\ DairyNZ Ltd., Newstead, Hamilton 3240, New Zealand
}

\section{ABSTRACT}

This study tested the hypothesis that a commercially available system for detecting estrus based on cow activity would perform similarly to that of typical, visual assessment of mounting indicators placed on the tail head of the cow. The hypothesis was applied to a large, pasture-grazed, seasonal-calving dairy herd, and the technology was tested as a stand-alone system. One of 2 types of commercially available collar-mounted activity meters was fitted to 635 cows, and the activity data collected during the 37-d artificial breeding period were analyzed. The first collar-mounted activity meter monitored activity only (AO collars), whereas the second meter measured activity and rumination characteristics (AR collars). Only activity data were used in the current study. Activity-based estrus alerts were initially identified using the default activity threshold value recommended by the manufacturer, but a range of activity threshold values was then analyzed to determine their effect on estrus detection performance. Milk progesterone data and insemination records were used to identify gold standard positive $(\mathrm{n}=835)$ and negative $(\mathrm{n}=22,660)$ estrus dates, to which activity alerts were compared. Visual assessment of mounting indicators resulted in a manual detection performance of $91.3 \%$ sensitivity (SN), $99.8 \%$ specificity (SP), and 94.5\% positive predictive value (PPV). The AR collars achieved 76.9, 99.4, and $82.4 \%$ for SN, SP, and PPV, whereas the AO collars achieved 62.4, 99.3, and $76.6 \%$ for SN, SP, and PPV, respectively. The observed performance of the activity systems may be underestimated due to test design and applied assumptions, including determining the date of estrus. Lowering the activity threshold from the default value improved sensitivity but the number of false positive alerts was considered to become unmanageable from a practical perspective as sensitivity reached peak values. Time window analysis, receiver operating characteristic curves, and curves of SN and PPV were found to be useful in the

Received September 14, 2011.

Accepted February 10, 2012.

${ }^{1}$ Corresponding author: claudia.kamphuis@dairynz.co.nz analysis and interpretation of results. They generate relevant performance data that allow for meaningful comparisons between similar studies. Although the 2 activity systems tested did not perform to the high level of manual estrus detection found in this study, the potential exists for these systems to be a valuable tool on farms with lower estrus detection performance or for farmers managing larger herds.

Key words: automated estrus detection, activity monitoring, performance evaluation, pasture-grazed seasonal dairying

\section{INTRODUCTION}

A high level of estrus detection is required to maximize productivity and profit in pasture-grazed, seasonal-calving dairy systems. Farm personnel usually detect cows in estrus by observing them for signs of estrus-related behaviors; for example, willingness to stand while being mounted (Eradus et al., 1992). Visual observation is typically aided by mounting indicators such as tail paint (MacMillan and Curnow, 1977). The tail paint method involves the application of a strip of enamel paint on the head of the tail. Visual observation of this paint strip being removed, broken, or obviously rubbed is then used as an estrus detection tool (MacMillan and Curnow, 1977). Achieving efficient estrus detection by visual observation requires experience, diligent attention, and time (Harris et al., 2010), but these requirements can be challenging because of increasing herd size and increasing reliance on unskilled labor (Anonymous, 2010; Newman, 2011). In addition, the modern, high-yielding dairy cow tends to have a lower intensity and shorter duration of estrus, making visual detection of estrus more difficult (Lopez et al., 2004; Harris et al., 2010). Accordingly, interest continues to increase in automated estrus detection systems (Cuthbert, 2008) to replace or aid this manual detection of estrus.

An increase in locomotive activity is a measure that can be used to predict estrus (Eradus et al., 1992), and activity meters (using pedometer or accelerometer technology) for automated estrus detection have been reported as useful. However, many of these studies were 
conducted with cows in housed systems (e.g., de Mol et al., 1997; At-Taras and Spahr, 2001; Roelofs et al., 2005). Extrapolation of the results to a pasture-grazed dairy system is questionable, because pasture-grazed cows typically have higher routine activity levels that may obscure increased activity related to estrus (Verkerk et al., 2001). Previous reports on the use of activity monitoring systems for estrus detection in pasture-grazed dairy systems have been experimental (Verkerk et al., 2001; McGowan et al., 2007; Sakaguchi et al., 2007), did not monitor activity for a complete artificial breeding period (Hockey et al., 2010), or focused on the potential benefits of adding other automatically retrieved data to improve detection performance, rather than studying the performance of activity monitoring alone in detecting estrus (Harris et al., 2010). Therefore, the detection performance of automated activity-based estrus detection systems when applied as a stand-alone system on large-scale, commercial, seasonal-calving, pasture-grazed dairy farms remains unknown.

The current study tested the hypothesis that a commercially available system for detecting estrus based on cow activity would perform similarly to that of typical visual assessment of mounting indicators placed on the tail head of the cow. The hypothesis was applied to a large, pasture-grazed, seasonal-calving dairy herd and the technology was tested as a stand-alone system. A time window analysis was conducted to assess sensitivity $(\mathbf{S N})$, specificity $(\mathbf{S P})$, and positive predictive value (PPV) of 2 types of collar-mounted activity meters. The tradeoffs between these performance measures were explored by altering activity threshold values and by widening the time windows in which activity-based estrus alerts were considered true positive.

\section{MATERIALS AND METHODS}

Approval was gained from the Ruakura Animal Ethics Committee before the start of the study. Data were collected at the Lincoln University Dairy Farm (LUDF) in Canterbury, New Zealand. The LUDF is a commercial demonstration farm, meaning that farm objectives, management policies, and animal performance measures are available to the scrutiny of farmers and rural professionals. The LUDF is an irrigated farm of 160 ha with approximately 670 cows (predominantly Jersey $\times$ Friesian crossbred) grazed on perennial ryegrass and clover pastures. The herd is milked twice daily (milking starts at 0500 and $1430 \mathrm{~h}$ ) through a 50-bail rotary milking parlor equipped with electronic identification and auto-drafting capability.

For this study, data were collected from $3 \mathrm{wk}$ before the planned start of mating on October 25, 2010, until the end of the 37-d artificial breeding period. The standard procedure at LUDF during the artificial breeding period was to detect cows in estrus by visually observing the degree of removal of the applied tail paint (MacMillan and Curnow, 1977) during the a.m. milking. In addition to this tail paint, a second mounting indicator (heat patches) was used on this farm in a parallel study. These heat patches were visible to farm staff when manually observing cows in estrus. When cows were observed to be in estrus, they were inseminated directly after the a.m. milking session. After the $37 \mathrm{~d}$ of artificial breeding, bulls were introduced into the herd for a further $33 \mathrm{~d}$ of natural mating. Pregnancy diagnoses were conducted on 2 separate occasions (January 10 and February 15, 2011) by the herd veterinarian using ultrasonography to age the fetuses for all pregnancies, as well as to identify cows that were not pregnant following the 10-wk mating period.

The average age of cows and the average DIM at the planned start of mating were $4.2 \pm 2.1 \mathrm{yr}$ and $62 \pm 18$ d; the average DIM at the first estrus date within the artificial breeding period was $73 \pm 19$ d. Average milk production during the $37-\mathrm{d}$ artificial breeding period declined from 2 to $1.6 \mathrm{~kg}$ of milksolids (protein plus fat yield)/cow per day. The interval between planned start of calving to planned start of mating was $62 \pm$ $18 \mathrm{~d}$. The proportion of the herd receiving at least one insemination during the first $21 \mathrm{~d}$ of the artificial breeding period was $87 \%$. The conception rate to first and second inseminations was $52 \%$ on each occasion. The proportion of the herd pregnant by $42 \mathrm{~d}$ of mating was $71 \%$, and the nonpregnancy rate at the end of the 10 -wk breeding period was $13 \%$.

\section{Activity Monitoring}

Two types of collar-mounted activity meters using accelerometer technology (SCR Engineering Ltd., Netanya, Israel) were fitted to 660 cows 3 wk before the start of the artificial breeding period. One activity meter measured activity only (AO collars), and the other measured activity and rumination characteristics (AR collars). Although the latter collars measured rumination characteristics, only the activity data were analyzed in this study. Assignment of collar types to the herd was balanced for age and breeding worth (an index of genetic merit used in New Zealand). Several cows were sold or removed from the herd before the planned start of mating; in the final outcome, activity data were available for 635 cows (320 cows with AO collars, and 315 with AR collars) during the $37 \mathrm{~d}$ of artificial breeding. Activity data were not available to the LUDF farm staff so that the manual estrus detection method was not influenced by activity measures. 
Both collar types make use of an accelerometer, an onboard data processing unit that analyses the movements of the cow using a complex algorithm, and memory that stores the resulted activity index in 12 two-hour blocks each day. Each time the cow walks under a collar reader (3 in the current study: the first was located above the milking platform near the entry, the second near the exit of the milking platform, and the third above the auto-weighing platform), data are sent via infrared communication from the collar to the farm computer (Heatime for PC, SCR Engineering Ltd., Netanya, Israel). Using a specially developed algorithm, a weighted activity index is calculated for each 2 -h time block, which expresses the momentary deviation of the activity (averaged over the last 8-12 h) from the average activity in the same period in the past $7 \mathrm{~d}$ (Bar, 2010). These weighted activity index data were used to generate activity alerts: if weighted activity in any of the 2-h time blocks exceeded a user-defined threshold value (default activity threshold value was 5.2 as recommended by the manufacturer), an activity alert was reported for that cow at the next milking.

\section{Milk Sampling for Determining Progesterone Levels}

Composite milk samples were collected weekly from all cows in the herd $(\mathrm{n}=666)$ during the 3 wk before the planned start of mating, and twice weekly during the 37-d artificial breeding period using LIC (Hamilton, New Zealand) standard equipment and herd-testing procedures. All collected milk samples were analyzed for progesterone content by a commercial laboratory (Gribbles Veterinary Pathology Ltd., Mosgiel, New Zealand) using a commercial RIA double-antibody kit in accordance with the manufacturer's instructions (ImmuChem, ICN Biomedical Inc., Costa Mesa, CA). Milk samples were mixed thoroughly before assay to reincorporate the cream. The interassay CV (20 assays) were $18.3,5.4$, and $5.6 \%$ for standard concentrations (Trilevel Lyphochek Immunoassay Plus Control, BioRad Laboratories, Hercules, CA) of 0.5, 8.2, and 20.7 $\mathrm{ng} / \mathrm{mL}$, respectively. Mean intraassay CV were 5.2 and $6.1 \%$ for milk samples having concentrations of 5.8 and $12.2 \mathrm{ng} / \mathrm{mL}$ (20 replicates per pool standard), respectively.

\section{Gold Standard Definition for Estrus}

To identify when cows were likely to have been in estrus, individual milk progesterone profiles were constructed for each cow. Trough-like curves in these progesterone profiles were assumed to indicate a periovulatory event (i.e., luteolysis followed by ovulation with subsequent development of the new, functional corpus luteum) and were initially located as evidence of an ovulation. The following rules were then used to assign an estrus date within this trough: (1) the estrus date was assigned as the day of AI where this resulted in a confirmed positive pregnancy outcome, regardless of the shape of the progesterone profile; (2) the estrus date was also assigned as the day of an unsuccessful AI when the insemination was coincident with the trough-like curve in progesterone concentrations; (3) if a trough-like curve occurred without a corresponding day of AI, the estrus date was set preferentially as the date with an activity alert, or (4) in the absence of an AI date and activity alert date, as being at the center of the trough-like curve in progesterone concentrations.

In total, 116 cows (17.4\% of the entire herd) ovulated for the first time after the planned start of mating. For these cases, the time of that ovulation was estimated to precede the elevation in progesterone following a prolonged period of basal milk progesterone. Specific rules around assigning a date of estrus to detect first ovulations were the same as described previously when the estimated day of first ovulation corresponded with a date of $\mathrm{AI}$ or an activity-based alert, but a date of estrus was not assigned when there was no supporting evidence that first ovulation was associated with a detectable behavioral estrus. This last rule was applied to be consistent with previous studies reporting that the majority of first ovulations are not accompanied by estrus in pasture-grazed systems (Burke et al., 1995; McDougall et al., 1995). Further assumptions made were (1) an AI was only conducted after visual detection of estrus, and (2) cows were always inseminated when an estrus was detected visually during the $37-d$ period of artificial breeding.

The assigned estrus dates were considered gold standard positives $\left(\mathbf{G S}_{\text {pos }}, \mathrm{n}=835\right.$ ), and those dates that were not assigned as an estrus date were considered gold standard negatives $\left(\mathbf{G S}_{\text {neg }}, \mathrm{n}=22,660\right)$. Because cows were inseminated only after a.m. milking, the $\mathrm{GS}_{\text {pos }}$ and $\mathrm{GS}_{\text {neg }}$ dates refer to a.m. milking sessions only.

\section{Matching Activity Alerts to Gold Standard Events}

To assess estrus detection performance of the activity collars, activity alerts were assigned to a.m. milkings retrospectively, applying the following rules and assumptions: (1) cows receiving an activity alert at the current a.m. milking or at the previous p.m. milking were assumed to be in estrus and to be inseminated directly after the current a.m. milking, and (2) activity alerts occurring at consecutive milkings were treated as one event until an occasion where a milking occurred 
without an activity alert. These assumptions effectively considered a 24-h time window (TW24-0) before a $\mathrm{GS}_{\text {pos }}$ a.m. milking in which an activity alert was expected. An activity alert was determined to be true positive (TP) when the alert corresponded with a $\mathrm{GS}_{\text {pos }}$ a.m. milking. Alerts were considered false positive (FP) when the alert corresponded with a $\mathrm{GS}_{\text {neg }}$ a.m. milking. The $\mathrm{GS}_{\text {pos }}$ a.m. milkings without an activity alert were considered false negative (FN) alerts, and $G S_{\text {neg }}$ a.m. milkings without an activity alert were considered true negative (TN) alerts (Table 1, Figure 1).

\section{Calculating Performance Indicators}

The 4 activity alert types (Table 1) were used to calculate 3 detection performance indicators. First, the $\mathrm{SN}$ was calculated as the number of $\mathrm{TP}$ alerts as a proportion of the total number of $\mathrm{GS}_{\text {pos }}$ events $[\mathrm{SN}=$ $100 \times \mathrm{TP} /(\mathrm{TP}+\mathrm{FN})]$. Second, SP was calculated as the number of $\mathrm{TN}$ alerts as a proportion of the total number of $\mathrm{GS}_{\text {neg }}$ events $[\mathrm{SP}=100 \times \mathrm{TN} /(\mathrm{TN}+\mathrm{FP})]$. Third, PPV was calculated as the number of TP alerts as a proportion of the total number of activity alerts $[\mathrm{PPV}=100 \times \mathrm{TP} /(\mathrm{TP}+\mathrm{FP})]$, representing the proportion of activity alerts that are likely to be correct.

\section{Exploring Different Time Windows}

In addition to the previously described TW24-0 time window, 2 other time windows were explored (Figure 1) to allow for a potential mismatch of setting $\mathrm{GS}_{\text {pos }}$ and $\mathrm{GS}_{\text {neg }}$ a.m. milkings (Sherlock et al., 2008). The second time window allowed an activity alert to be valid within a period of $48 \mathrm{~h}$ preceding a $\mathrm{GS}_{\text {pos }}$ a.m. milking (TW48-0). The third time window allowed an activity alert to be valid within a period of $48 \mathrm{~h}$ preceding and $24 \mathrm{~h}$ following a $\mathrm{GS}_{\mathrm{pos}}$ a.m. milking (TW48-24).

\section{Exploring Tradeoffs Between Detection Performance Indicators}

The trade-off between SN and SP was explored using receiver operating characteristic (ROC) analysis. This analysis plots $\mathrm{SN}$ on the y-axis and the FP rate (equivalent to $100 \%$ - SP) on the $\mathrm{x}$-axis (Fawcett, 2006) over the range of possible threshold values (Detilleux et al., 1999). The lower left point of the graph $(0,0)$ represents a point where the chosen threshold is so high that no alerts will be assigned, causing $\mathrm{SN}$ and $\mathrm{FP}$ rate to be $0 \%$ (and SP to be 100\%). The upper right point of the graph $(100,100)$ represents a point where the chosen threshold is so low that alerts are assigned to all instances, causing the SN and FP rate to be $100 \%$ (and SP to be 0\%). The ROC graph in this study was restricted to a maximum $\mathrm{FP}$ rate of $3 \%$ (equivalent to a SP of $\geq 97 \%$ ). The effects of changing the activity threshold value on the relationship between SN and PPV were explored by plotting SN and PPV levels against varying threshold values on the $\mathrm{x}$-axis.

\section{Data Analysis}

All 635 cows fitted with activity collars were in the milking herd for the entire artificial breeding period. This resulted in a total of 23,495 $\mathrm{d}$ and 281,940 twohour time blocks. In total, 25,837 two-hour time blocks $(9.2 \%)$ had missing weighted activity index values. Missing data could have occurred for various reasons, including collars not being read properly by the infrared readers. Because of a power failure at the planned start of mating, no activity data were recorded for a period starting at $1400 \mathrm{~h}$ on October 24 until $0200 \mathrm{~h}$ on October 26 ( $\mathrm{n}=10,951$ two-hour time blocks with missing activity data; $3.9 \%$ of the total two-hour time blocks present in the study, and $42.4 \%$ of the total two-hour time blocks with missing values). Within this period, data were missing for all 635 cows starting at $1600 \mathrm{~h}$ on October 24 until $2200 \mathrm{~h}$ on October 25. This meant that activity alerts for the a.m. milking on October 25 were not based on a 24 -h period of two-hour time blocks, but on an 8-h period of two-hour time blocks, and that alerts for the a.m. milking on October 26 were based on a 10-h period of two-hour time blocks with activity data.

Data preparation and calculation of performance indicators were performed using SAS (version 9.2, SAS Institute Inc., Cary, NC). The Chi-squared test (PROC FREQ in SAS) was used to test whether collar types were statistically significantly different in detecting estrus within each time window, assuming that each $\mathrm{GS}_{\text {pos }}$ event was independent. The $95 \%$ CI of performance indicator estimates were calculated in Excel (Office 2007, Microsoft Corp., Redmond, WA).

\section{RESULTS}

Manual detection resulted in $91.3 \% \mathrm{SN}, 99.8 \%$ SP, and $94.5 \%$ PPV. Activity collars (AR and AO together) had a lower detection performance with $59.8 \% \mathrm{SN}$, $99 \% \mathrm{SP}$, and $68.2 \% \mathrm{PPV}$ (Table 2). These data were based on the default activity threshold value of 5.2 and the strictest time window (TW24-0). The wider time windows (TW48-0 and TW48-24) showed improved detection performance, with TW48-24 having the highest overall performance (Table 2). Using TW48-24, the average number of 2 -h time blocks that exceeded the activity threshold was 5.5 for TP alerts (ranging from 1 to 12 ) and 2.2 for FP alerts (ranging from 1 to 11 ). 
Table 1. A $2 \times 2$ contingency table used for calculating sensitivity (SN), specificity (SP), and positive predictive value (PPV)

\begin{tabular}{llll}
\hline & & \multicolumn{2}{c}{ Gold standard } \\
\cline { 2 - 3 } Activity collar & $\begin{array}{l}\text { Positive } \\
\text { (estrus date) }\end{array}$ & $\begin{array}{l}\text { Negative } \\
\text { (no estrus date) }\end{array}$ & Performance indicator \\
\hline $\begin{array}{l}\text { Alert } \\
\text { (activity exceeds threshold) }\end{array}$ & True positive $(\mathrm{TP})$ & False positive $(\mathrm{FP})$ & $\mathrm{PPV}=\mathrm{TP} /(\mathrm{TP}+\mathrm{FP})$ \\
$\begin{array}{l}\text { No alert } \\
\text { (activity does not exceed threshold) }\end{array}$ & False negative $(\mathrm{FN})$ & True negative $(\mathrm{TN})$ & \\
\begin{tabular}{l} 
Performance indicator \\
\hline
\end{tabular} & $\mathrm{SN}=\mathrm{TP} /(\mathrm{TP}+\mathrm{FN})$ & $\mathrm{SP}=\mathrm{TN} /(\mathrm{TN}+\mathrm{FP})$ & \\
\hline
\end{tabular}

Time window TW24-0

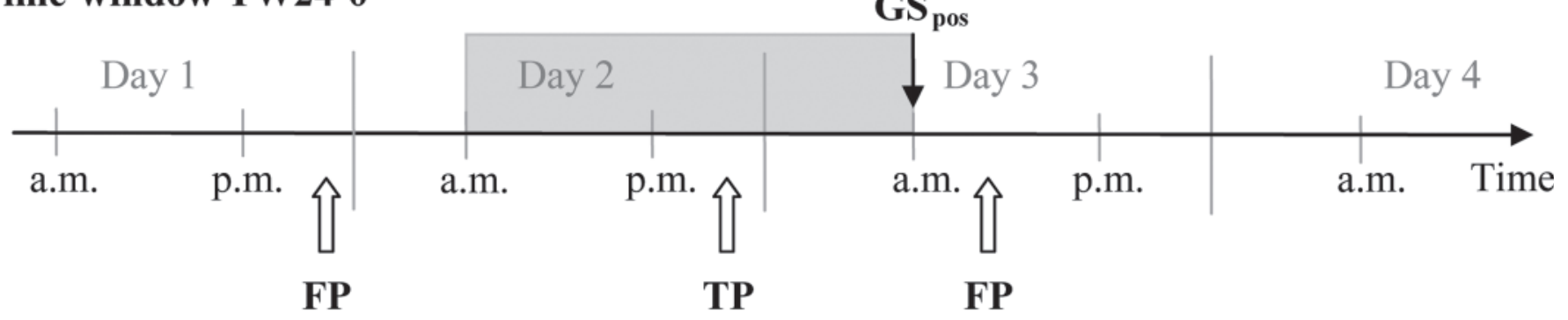

\section{Time window TW48-0}

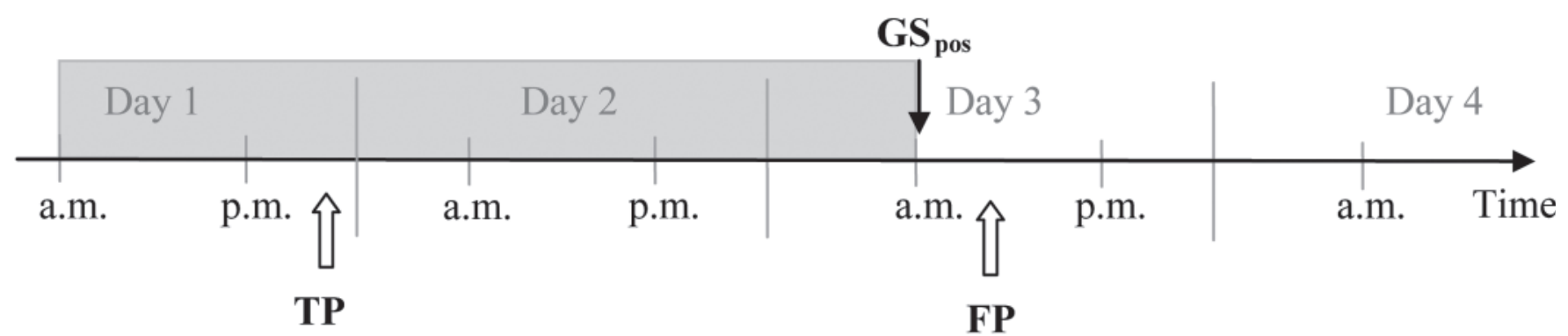

\section{Time window TW48-24}

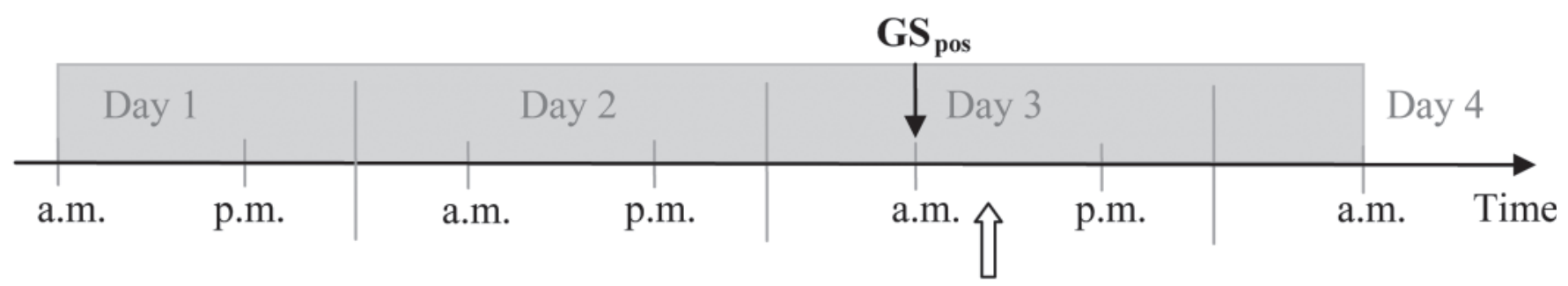

TP

Figure 1. Methodology for assigning true positive (TP) and false positive (FP) activity alerts (upward-pointing white arrows) using 3 time windows (TW). TW24-0 required an activity alert to fall within a 24-h time period (gray shaded area) preceding a gold standard positive (GS pos $_{\text {) }}$ a.m. milking to be considered TP, and any activity alert outside this 24-h time window was considered FP; TW48-0 required an activity alert to fall within a 48-h time period preceding a GS pos a.m. milking to be considered TP, and activity alerts outside this 48-h time window were considered FP; TW48-24 required an activity alert to fall within a 48-h time period preceding or a 24-h time period after a GS to be considered TP and, again, alerts outside this time window were considered FP. 
Table 2. Detection performance indicators sensitivity (SN), specificity (SP), and positive predictive value (PPV) calculated at the default threshold (5.2) during the 37-d artificial breeding period ${ }^{1,2}$

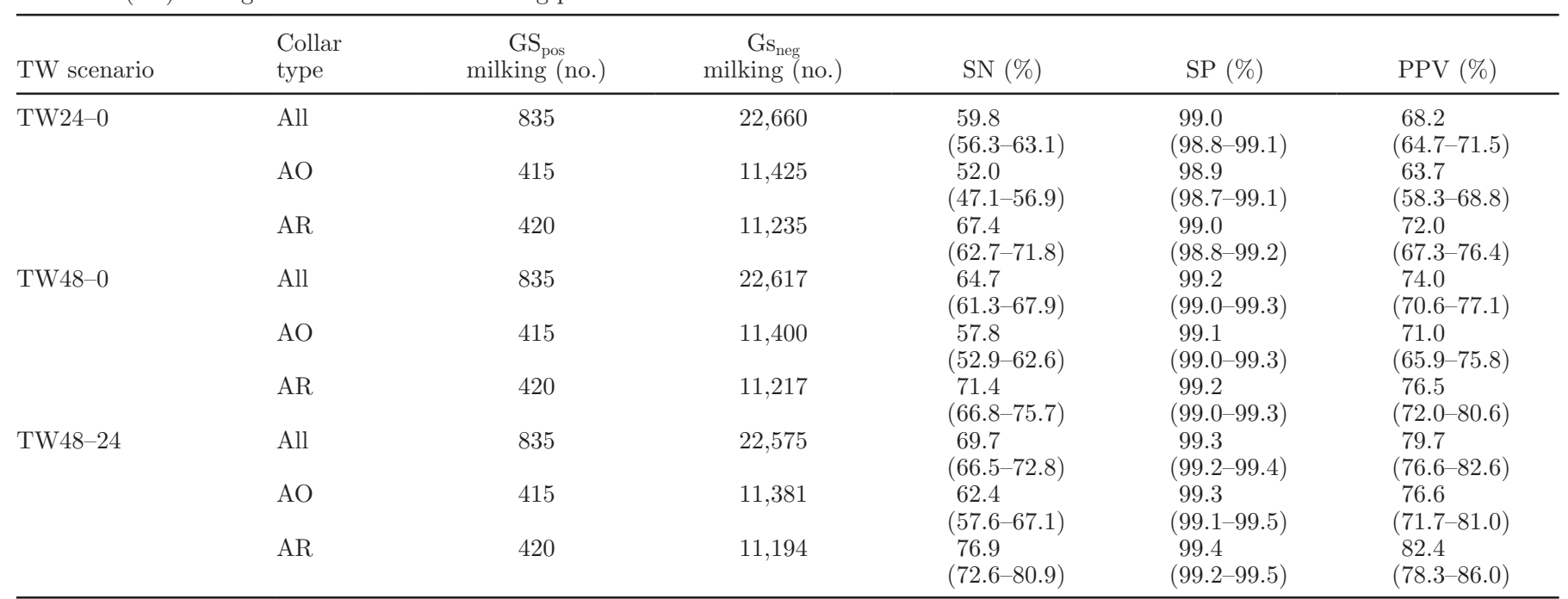

\footnotetext{
${ }^{1}$ Detection performance indicators (95\% CI in parentheses) are presented for collar types combined (All), collars measuring activity only (AO), and collars measuring activity and rumination characteristics (AR).

${ }^{2}$ Three time windows (TW) were analyzed: TW24-0 required an activity alert to fall within a 24 -h period preceding a gold standard positive $\left(\mathrm{GS}_{\mathrm{pos}}\right)$ a.m. milking to be a true positive alert; TW48-0 required an activity alert to fall within a 48-h time period preceding a GS pos a.m. milking to be a true positive alert; and TW48-24 required an activity alert to fall within a 48-h time period preceding or a 24 -h time period after a $\mathrm{GS}_{\text {pos }}$ a.m. milking. For each time window and collar type, the number of $\mathrm{GS}_{\text {pos }}$ a.m. milkings and gold standard negative $(\mathrm{GS}$ neg $)$ a.m. milkings used to calculate the detection performance indicators are reported.
}

When collar types were analyzed separately at the default activity threshold value, AR collars outperformed AO collars $(P<0.0001)$ for all 3 time windows in terms of SN (Table 2). In addition, the PPV of AR collars was higher $(P=0.016)$ than that of AO collars for TW24-0 (Table 2) and approached significance for TW48-24 ( $P$ $=0.053)$. The best detection performance was observed using the AR collars and TW48-24 (Table 2), but was lower than manual detection of estrus.

Because SN significantly differed between AR and $\mathrm{AO}$ collars, further results for the collar types are reported separately. Figure 2 displays 2 ROC curves, one for each collar type when using TW24-0, and demonstrates the tradeoff between SN and SP. As SN increases, an accompanying increase in FP alerts occurs, resulting in a decrease in SP. Within a $\mathrm{FP}$ rate range of $\leq 3 \%$ (or $\mathrm{SP}$ of $\geq 97 \%$ ), neither collar type could achieve SN of $\geq 90 \%$. Sensitivity was similar for both collar types, with a very low rate of $\mathrm{FP}(<0.3 \%)$. Beyond that point, the curves and the $95 \%$ CI bands showed that AR collars were more sensitive in detecting estrus than the AO collars. Figure 2 also shows that the default activity threshold value was at different points on the ROC curves for $\mathrm{AO}$ and AR collars. At a fixed $\mathrm{FP}$ rate of $1 \%$ (SP of $99 \%$ ), SN levels were 49.9 and $67.5 \%$ for $\mathrm{AO}$ and $\mathrm{AR}$ collars, respectively. At a fixed $\mathrm{SN}$ of $50 \%$, the FP rate was $1 \%(\mathrm{SP}=99 \%)$ for $\mathrm{AO}$ collars and $0.3 \%(\mathrm{SP}=99.7 \%)$ for AR collars. When using
TW48-0 and TW48-24, the ROC curves for both collar types showed similar trends, as presented in Figure 2, but the curves were at higher SN levels. At a fixed FP rate of $1 \%(\mathrm{SP}=99 \%)$, SN levels for AO collars were $60.1 \%$ at TW48- 0 and $65.9 \%$ at TW48-24. At the same FP rate, AR collars had a SN of $73.6 \%$ at TW48-0 and $79.4 \%$ at TW48-24.

The effect of changing the activity threshold value and time windows on SN and PPV for AO and AR collars is depicted in Figures 3 and 4. Again, neither collar type achieved a detection performance comparable to that of manual detection ( $\sim 90 \%$ SN with $94.5 \%$ PPV). Both collar types showed better detection performance (both SN and PPV increased) when time windows were wider. This increase was more pronounced when comparing TW24-0 with TW48-0 than when comparing TW48-0 with TW48-24. Changing time windows especially influenced SN at lower activity threshold values. When using TW48-24, changes in activity threshold values had a large effect on PPV for both collar types; for example, PPV was approximately $21 \%$ at an activity threshold of 2 and approximately $95 \%$ at an activity threshold of 10. The effect on SN was less pronounced but still apparent: SN was 86.5 and $88.6 \%$ for AO and AR collars, respectively, at an activity threshold of 2 and decreased to 44.8 and $63.8 \%$ for $\mathrm{AO}$ and AR collars, respectively, at a threshold value of 10 . The AR collars achieved the highest SN level (91\%) when the 


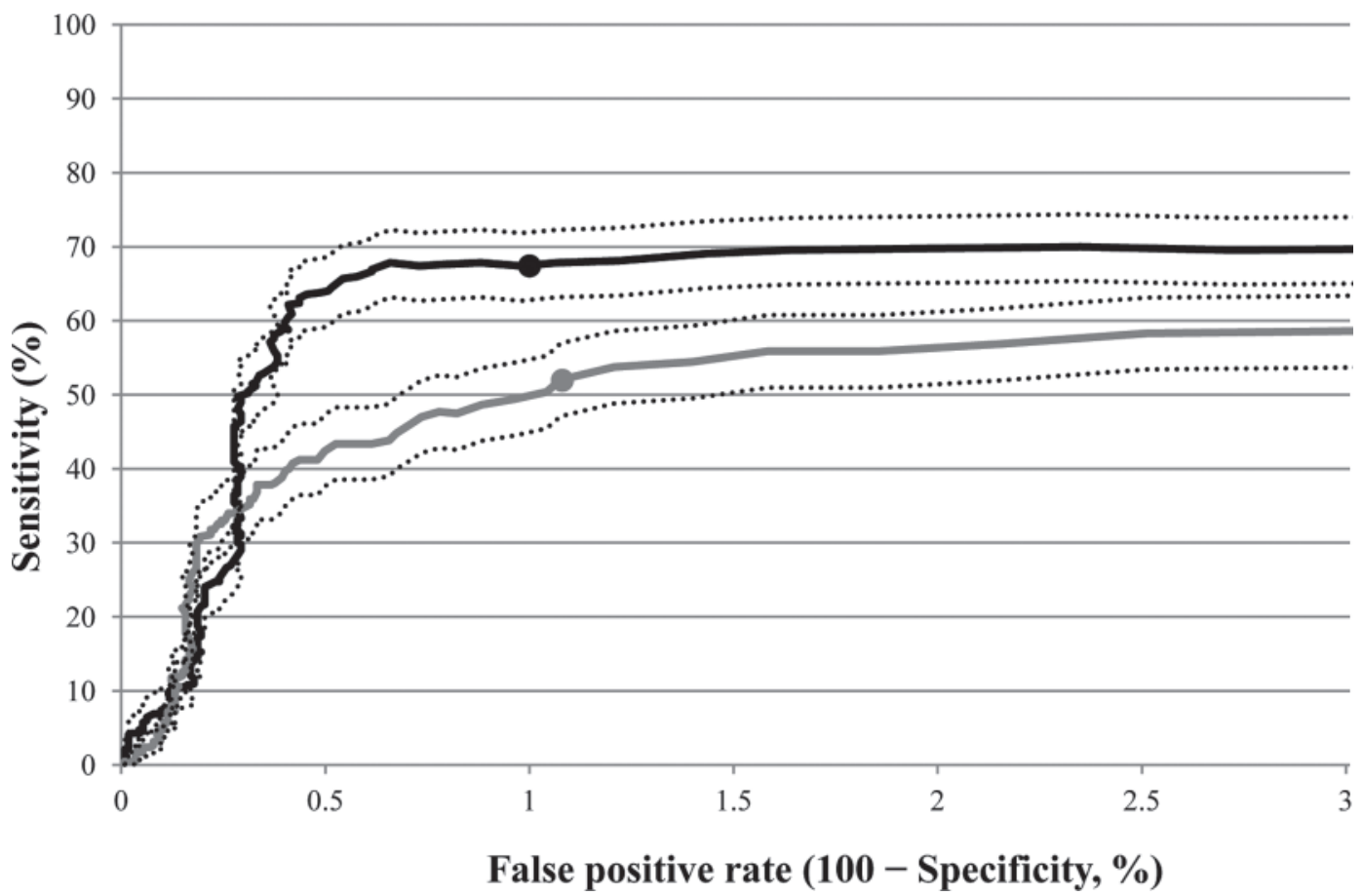

Figure 2. Receiver operating characteristic (ROC) curves (restricted to a maximum false positive rate of $3 \%$, equivalent to a minimum specificity of $97 \%$ ) and their $95 \%$ CI bands represented by dashed lines (.....): The gray line is for collars measuring activity only, the black line is for collars measuring activity and rumination characteristics. The curves are based on time window TW24-0, in which an activity alert was considered true positive when it occurred within $24 \mathrm{~h}$ preceding a gold standard positive a.m. milking. The dots (-) on each ROC curve represent detection performance at the manufacturer's recommended default threshold value of 5.2.

threshold value was lowered to 1.6 using TW48-24 but PPV decreased dramatically to $16.1 \%$. This implies that in practice, these collars could detect $90 \%$ of all estrus events during the artificial breeding period, but fewer than one-fifth of cows identified would truly be in estrus. Increasing the activity threshold value resulted in a higher PPV and a decreased SN. That is, fixing the PPV at $80 \%$ by changing the activity threshold value to 5.6 and 5.0 for $\mathrm{AO}$ and $\mathrm{AR}$ collars, respectively, resulted in $\mathrm{SN}$ of 60.5 and $77.4 \%$ for $\mathrm{AO}$ and $\mathrm{AR}$, respectively. When PPV was $80 \%$, there would be greater confidence that a higher proportion of cows identified were truly in estrus.

At the start of the artificial breeding period, a power failure resulted in consecutive 2 -h time blocks with missing activity data. These missing data may have affected the weighted activity index for the following 7 d. Excluding the first week from the artificial breeding period and beginning the performance analysis at the morning milking from November 2 using TW48-24 and the default activity threshold value of 5.2 resulted in SN, SP, and PPV of $62.2,99.2$, and $72.3 \%$ for AO collars (307 $\mathrm{GS}_{\mathrm{pos}}$ events) and SN, SP, and PPV of 74.6, 99.4, and $80.4 \%$ for AR collars (303 GS $\mathrm{S}_{\text {pos }}$ events). These are comparable levels to those reported in Table 2 , in which the whole artificial breeding period was considered.

To be confident that the $\mathrm{GS}_{\text {pos }}$ dates were assigned correctly to cases where an insemination failed to result in pregnancy, the performance indicators (using the default activity threshold value) were determined for 2 groups of cows: those confirmed pregnant to their first AI $(\mathrm{n}=317)$ and those that did not conceive to first AI $(\mathrm{n}=283)$. Overall SN at TW24-0 was 63.9 and $64.3 \%$ for confirmed pregnant and nonpregnant cows, respectively. For TW48-0, SN was 67.6 and $68.4 \%$ for pregnant and nonpregnant cows, respectively, whereas for TW48-24, SN was $73.7 \%$ for both groups.

In total, 116 cows $(17.4 \%$ from all 666 cows in the herd) had their first ovulation (based on progesterone profiling) after the planned start of mating. Of these, 43 cows had a $\mathrm{GS}_{\text {pos }}$ date assigned to that ovulation, and 27 of them received an activity alert using TW24-0 $(\mathrm{SN}=62.8 \%)$.

On average, at the default activity threshold value, 19 cows received an activity alert on a daily basis (TP and FP activity alerts) during the artificial breeding period. Of these 19 alerts, 6 (32\%) were FP. However, excessive numbers of FP alerts (ranging between 14 and 


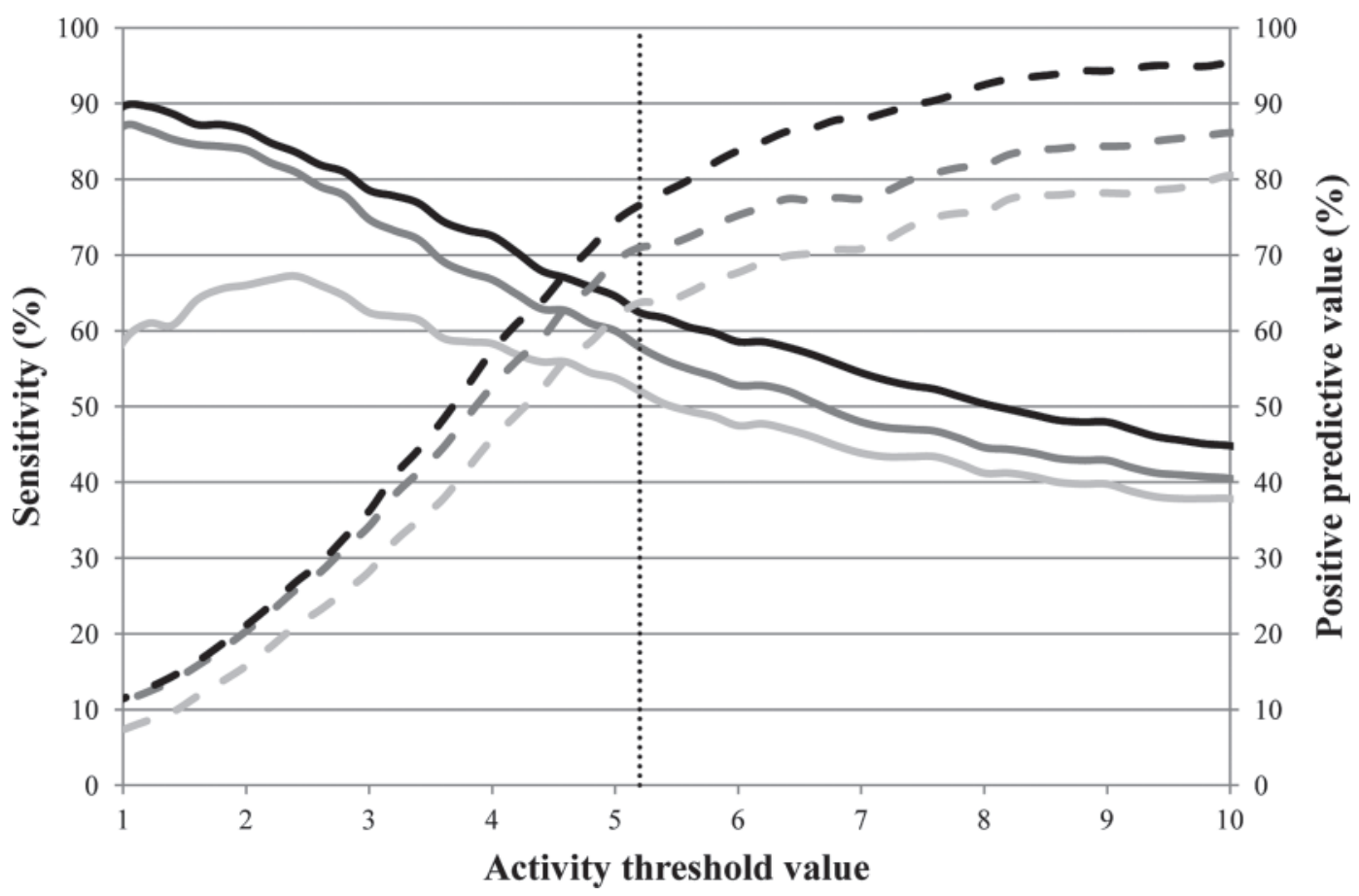

Figure 3. Effect of changing the activity threshold value (x-axis) on sensitivity (y-axis) and positive predictive value (second y-axis) for collars measuring activity only for 3 time windows; TW24-0, in which an activity alert was only considered true positive when it occurred within $24 \mathrm{~h}$ preceding a gold standard positive ( $\mathrm{GS}_{\text {pos }}$ ) a.m. milking (represented by light gray lines); TW48-0, in which an activity alert was considered true positive when it occurred within $48 \mathrm{~h}$ preceding a GS pos a.m. milking (represented by dark gray lines); and TW48-24, in which an activity alert was considered true positive when it occurred within $48 \mathrm{~h}$ preceding and $24 \mathrm{~h}$ after a $\mathrm{GS}_{\text {pos }}$ a.m. milking (represented by black lines). Curves for sensitivity are represented by solid lines and curves for positive predictive value by dashed lines. The default activity threshold value is represented by the vertical dotted line.

$35 \mathrm{FP}$ alerts) occurred at 5 morning milkings. When data from these $5 \mathrm{~d}$ were excluded from the analysis, detection performance (based on 358 GS $_{\text {pos }}$ events analyzed with TW48-24 and the default activity threshold value) for AO collars was $62,99.7$, and $88.4 \%$ for SN, $\mathrm{SP}$, and PPV, respectively. Detection performance for cows with an AO collar that were confirmed pregnant to first AI (142 $\mathrm{GS}_{\text {pos }}$ events) was 60.6, 99.7, and 86.9\% for SN, SP, and PPV, respectively. Excluding these 5 $\mathrm{d}$, detection performance using TW48-24 (377 GS $_{\text {pos }}$ events) and the default activity threshold value for the AR collars was $75.6,99.7$, and $90.8 \%$ for SN, SP, and PPV, respectively. For cows with an AR collar that were confirmed pregnant to first AI (141 GS $\mathrm{p}_{\mathrm{pos}}$ events), detection performance was $87.2,99.8$, and $91.8 \%$ for $\mathrm{SN}, \mathrm{SP}$, and PPV, respectively.

\section{DISCUSSION}

This study demonstrated that the activity meters evaluated were unable to perform to the same level as manual detection of estrus when used as a stand-alone system in this particular dairy herd (a large, pasturegrazed, seasonal-calving, commercial farm). This finding applied to all time windows (Table 2) and all activity thresholds analyzed (Figures 2, 3, and 4). Excluding the first week of the artificial breeding period (because of a power outage resulting in missing activity data) did not change this result. The AO collars did not perform as well as the AR collars within any time window analyzed (Table 2). The difference in detection performance between collar types may be explained by the inclusion of faulty AO collars. It was not, however, possible to confirm whether this had happened. The results from this study indicate the need for a system that automatically identifies faulty collars when in use on-farm.

For automated detection systems to be of practical use in seasonal-calving, pasture-grazed herds, they should perform at least to the level achieved by the average farmer using manual detection methods. The manual assessment using both tail paint and heat patches to detect estrus events showed a very high level of performance on this farm, as progesterone-defined estrus events $\left(\mathrm{GS}_{\text {pos }}\right.$ events) were identified with $91.3 \%$ SN, 99.8\% SP, and 94.5\% PPV using the strictest time window (TW24-0). Diskin and Sreenan (2000) suggested that $90 \%$ is the highest $\mathrm{SN}$ achievable when 


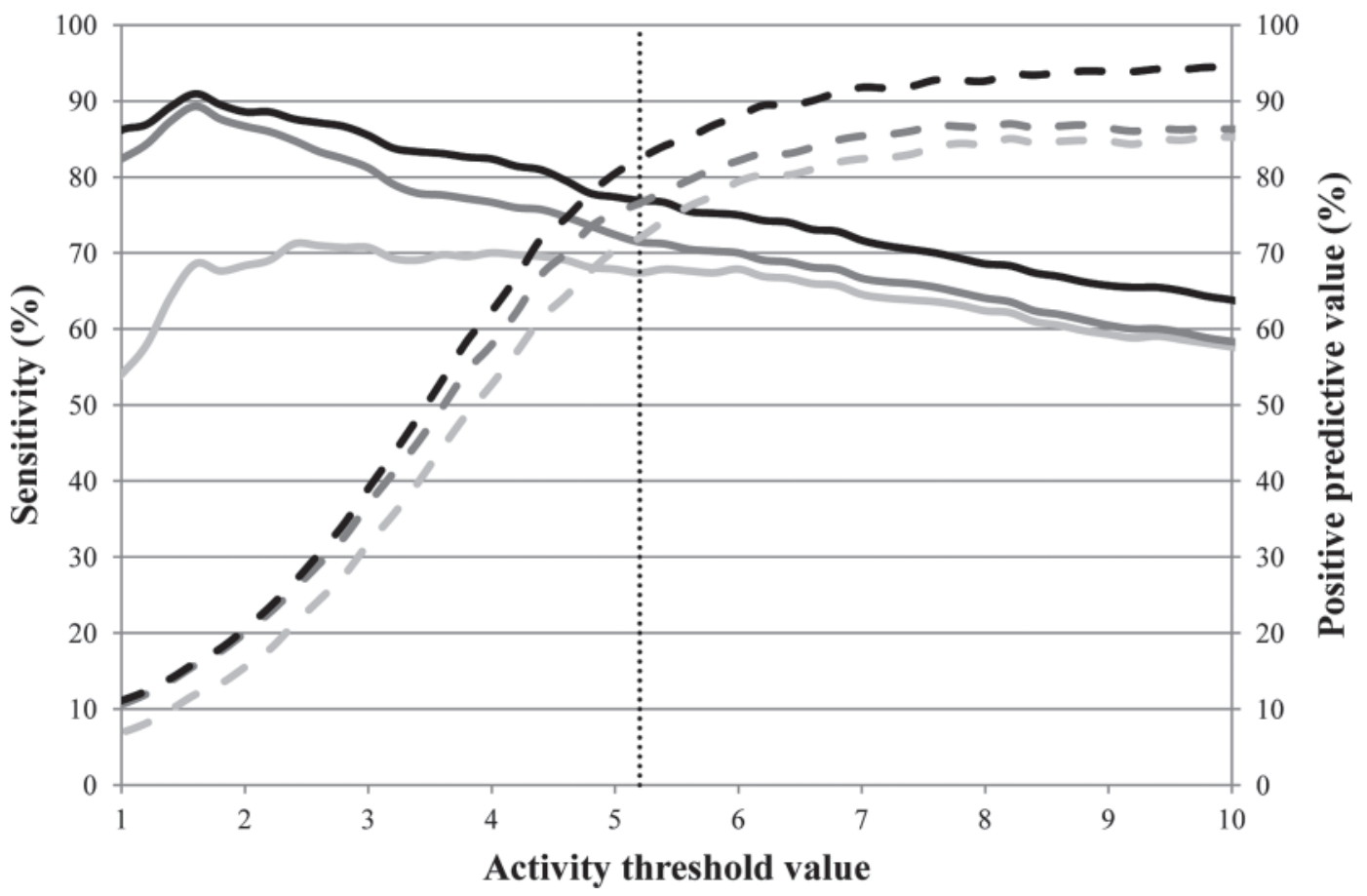

Figure 4. Effect of changing the activity threshold value (x-axis) on sensitivity (y-axis) and positive predictive value (second y-axis) for collars measuring activity and rumination characteristics for 3 time windows; TW24-0, in which an activity alert was only considered true positive when it occurred within $24 \mathrm{~h}$ preceding a gold standard positive $\left(\mathrm{GS}_{\mathrm{pos}}\right)$ a.m. milking (represented by light gray lines); TW48-0, in which an activity alert was considered true positive when it occurred within $48 \mathrm{~h}$ preceding a $\mathrm{GS}_{\text {pos }}$ a.m. milking (represented by dark gray lines); and TW48-24, in which an activity alert was considered true positive when it occurred within $48 \mathrm{~h}$ preceding and $24 \mathrm{~h}$ after a GS ${ }_{\text {pos }}$ a.m. milking (represented by black lines). Curves for sensitivity are represented by solid lines and curves for positive predictive value by dashed lines. The default activity threshold value is represented by the vertical dotted line.

using tail paint as an estrus detection aid. The visual assessment of heat patches in addition to the standard procedure of assessing tail paint may have contributed to greater detection performance. However, as reproduction performance indicators were similar compared with that in previous years, this effect was assumed to be minor. Studies of pasture-grazed systems have reported SN of visual observation, aided by tail paint, ranging from $71.6 \%$ (Verkerk et al., 2001) to more than 92\% (Xu et al., 1998; McGowan et al., 2007). These 3 studies were conducted with small numbers of cows, and estrus events occurred under controlled, experimental conditions. Taking these reported sensitivity levels into account, a performance level of $80 \% \mathrm{SN}$ with $80 \%$ PPV could be considered average performance and a suitable minimum performance target for automated estrus detection systems. The AR collars analyzed in this study were able to achieve a SN of $78 \%$ when used as a stand-alone system by lowering the activity threshold value (Figure 4). At this SN level, SP would be $99 \%$ and PPV would be $78 \%$. This performance level may be of benefit for farmers who have a lower level of manual detection (e.g., <80\%) than indicated for the current study. In addition, the technology may appeal to farm- ers managing larger herds ( $>500$ cows) as such herds rely more on inexperienced staff and handle a higher number of cows per person (Newman, 2011).

This study retrospectively evaluated the performance of activity collars in detecting estrus events. To do this, it was necessary to apply several assumptions. These assumptions, however, may not always reflect real-time use of these collars and may have resulted in lower performance rates than would be expected in practice. One assumption made in this study was that all ovulations beyond the first ovulation after calving were accompanied by detectable estrus behavior. This necessary assumption may have resulted in setting some $\mathrm{GS}_{\mathrm{pos}}$ dates where ovulation was not accompanied by a detectable estrus, which in turn may have led to underestimating detection performance of both manual and activity-based detection systems. Previous reports in pasture-grazed dairy systems have indicated that 11 to $13 \%$ of second ovulations and 0 to $17 \%$ of third ovulations are not accompanied by detectable estrus behavior (McDougall, 1994; Burke et al., 1995). Another assumption was that activity meters were used as a stand-alone system to detect cows in estrus. This meant that activity alerts were the only information 
used to test the performance of the activity-based system. As a consequence, the assumption was that all cows receiving an activity alert were in estrus and that they were inseminated at the a.m. milking after they exceeded the activity threshold value for the first time. This assumption, however, may have resulted in a lower PPV than would be seen in practice. In practice, cows that receive an activity alert are usually confirmed to be in estrus by a secondary test (e.g., visual inspection of the alerted cow or inspection of previous activity alerts and insemination records) conducted by the farm staff. This secondary test can help to reduce FP alerts, which in turn will increase PPV. In the current study, 5 separate days during the artificial breeding period had an uncharacteristically high number of FP alerts. When these 5 dates were excluded, PPV increased from 76.6 to $88.4 \%$ for AO collars and from 82.4 to $90.8 \%$ for AR collars for the TW48-24 time window and the default activity threshold value. No explanation could be found for these $5 \mathrm{~d}$ with uncharacteristically high numbers of FP alerts; for example, unusual management tasks or significant earthquakes (the LUDF farm is situated in the Canterbury region, an area where significant earthquakes occurred during the testing period), but it would be reasonable to assume that, in practice, users of this technology would be able to identify at least some of these alerts as FP when they occur on specific occasions. The assumption that cows received an alert after they exceeded the activity threshold for the first time may also have resulted in a lower PPV. Altering this assumption into one that assigned activity alerts to cows after multiple 2-h time blocks exceeded the activity threshold could have resulted in a higher PPV but to the detriment of SN, because there were TP alerts based on one 2-h block that exceeded the activity threshold.

The SN of activity collar data for cows confirmed pregnant to first AI was similar to that of cows confirmed not pregnant to the first AI. This outcome provides confidence in the rules applied to set $\mathrm{GS}_{\text {pos }}$ dates in the absence of a confirmed pregnancy, but a degree of inaccuracy may have been incurred in setting the $\mathrm{GS}_{\text {pos }}$ if the reason for an unsuccessful AI was incorrect timing. This means that, within TW24-0, an activity alert may have been considered as a FP but could have been a TP alert if the true ovulation time was a day earlier or later than the $\mathrm{GS}_{\mathrm{pos}}$ date assigned. To explore this potential mismatch, 2 additional time windows were considered (TW48-0 and TW 48-24). Detection performance improved as time windows became wider (Table 2). This trend was also observed for cows that were confirmed pregnant to first AI, which was unexpected as their ascribed $\mathrm{GS}_{\mathrm{pos}}$ dates had a very high likelihood of being correct. This raises the question as to which time window is the most appropriate for reporting detection performance. Both collar types showed an increase in SN of approximately $5 \%$ between TW24-0 and TW48-0, and a further $5 \%$ between TW48-0 and TW48-24 (Table 2). More detailed analysis of the 2-h time block activity data revealed 41 additional TP alerts as the time window increased from TW24-0 to TW48-0. Among these, 37 cases had a second activity alert in the 24-h time period before the $\mathrm{GS}_{\text {pos }}$ date, and $86.4 \%$ of these were on a plane of rising activity, with the second alert having a higher activity value than the first alert. These second alerts were ignored in the initial analysis due to the assumption that cows would be inseminated at the a.m. milking after exceeding the activity threshold the first time. Of these 39 cases, 24 exceeded the activity threshold for the first time during the 2-h time block running from 24 to 26 $\mathrm{h}$ before the $\mathrm{GS}_{\text {pos }}$ date; that is, occurring just outside the 24-h time window. This interpretation is supported by reports by Roelofs et al. (2005) and Hockey et al. (2010), who found that the onset of increased activity began approximately $30 \mathrm{~h}$ before ovulation. Cows approaching estrus are often restless, show increasing walking, trail other cows, and bellow more (Diskin and Sreenan, 2000). Extending the time window further (from TW48-0 to TW48-24) produced another 42 TP alerts. Of these, $26(61.9 \%)$ had exceeded the default threshold value for the first time within a 2 -h period following the $\mathrm{GS}_{\text {pos }}$ event, and 35 (83.3\%) had exceeded it within a 4-h period following the $\mathrm{GS}_{\mathrm{pos}}$ event. Altogether, these figures demonstrate the effect of the assumption that cows were in estrus after exceeding the activity threshold value the first time. The TW48-24 time window appears to be suitable for calculating detection performance for the activity collars $(\mathrm{SN}=$ $62.4 \%$ for AO collars and $\mathrm{SN}=76.9 \%$ for AR collars at the default threshold value, Table 2).

This study applied 2 methods to report detection performance of the activity collars. The first method (ROC graph, Figure 2) showed the tradeoff between SN and SP by varying the activity threshold values. The ROC curves were arbitrarily limited to a maximum FP rate of $3 \%$ (i.e., minimum SP of $97 \%$ ). This $\mathrm{FP}$ rate would generate an average of $18 \mathrm{FP}$ alerts per day during the $37 \mathrm{~d}$ of artificial breeding in a herd of about 650 cows. The ROC curves have the advantage that both measures used to generate the curve (SN and SP) are insensitive to changes in prevalence (Fawcett, 2006) and, therefore, the ROC curves will not change even if the proportion of positive and negative instances were to change. In addition, ROC curves are useful in comparing detection performance between detection systems (Fawcett, 2006), even when these systems use different activity measures. The disadvantage, however, 
is that ROC curves may be difficult for end-users to interpret and the graphs do not provide direct information about the activity threshold values used to create them. As a practical alternative, the second method of reporting performance (plotting SN and PPV against the chosen activity threshold value) has the advantage that changes in detection performance due to changes in activity thresholds are easy to interpret. In addition, the PPV presents a more direct measure of the proportion of activity alerts that are likely to be correct (Sherlock et al., 2008). The disadvantage is that PPV can be influenced by many factors, including SN and SP (Hockey et al., 2010), which makes it more difficult to compare PPV across farms and studies; therefore, this performance indicator should be used with care.

Several previous reports have evaluated the estrus detection performance of activity meters. Some were experimental and reported only SN and PPV, which makes a comparison difficult for the reasons described above (PPV being prevalence dependent). de Mol et al. (1997) analyzed detection performance of a legmounted pedometer and reported $80.9 \%$ SN and $98 \%$ SP using a time window comparable to the TW48-24 applied in this study. Performance was thus comparable to the results of the current study although the study reported by de Mol et al. (1997) involved cows housed indoors. Verkerk et al. (2001) reported $46.9 \% \mathrm{SN}$ and 95.4\% SP for collar-mounted activity meters used in a pasture-based system. At that same SP level, SN in the current study was 61.8 and $69.3 \%$ for $\mathrm{AO}$ and $\mathrm{AR}$ collars, respectively, using TW24-0. A larger study on detection performance of neck-mounted activity collars in a pasture-grazed system was conducted by Hockey et al. (2010). They reported $90.4 \%$ SN, $98.2 \%$ SP, and $75.8 \%$ PPV, higher than that found for either collar in the current study, but the analyses used a wider time window (approximately $5 \mathrm{~d}$ ), which may explain the differences in detection performance. These examples indicate the need for a more consistent method of reporting and comparing the detection performance of activity meters used for estrus detection.

\section{CONCLUSIONS}

The collar-mounted activity meters did not perform to the same level of manual detection when applied as a stand-alone estrus detection system. However, the reported detection performance may be below that expected in practice, due to test design and the assumptions made. These systems may still be of value on farms with lower manual estrus detection performance than indicated in this study or for farmers managing larger herds. This study demonstrated the effects of changing threshold values and time windows on detec- tion performance and used ROC graphs and SN and PPV curves to show these effects. These approaches may allow for more meaningful comparisons between studies of activity meters for estrus detection.

\section{ACKNOWLEDGMENTS}

We gratefully acknowledge P. Edwards (DairyNZ Ltd., Newstead, New Zealand) and N. Purdie (DairyNZ Ltd., Lincoln, New Zealand) for their technical assistance with this study and the LUDF farm staff for their assistance with data collection. Also gratefully acknowledged is B. Dow (DairyNZ Ltd., Newstead, New Zealand) for assisting with the statistical analyses. This project was supported in kind by Milfos International Ltd. (Hamilton, New Zealand) and the South Island Dairying Development Centre (SIDDC). It was supported financially through the MAF Sustainable Farming Fund (Project 10/024), through DairyNZ Inc. (Project AN1004 and Project SY1006) and through the South Island Dairy Event (SIDE).

\section{REFERENCES}

Anonymous. 2010. New Zealand Dairy Statistics. Accessed May 2 2011. http://www.dairynz.co.nz/page/pageid/2145868118/New_ Zealand_Dairy_Statistics_2009-10. htm.

At-Taras, E. E., and S. L. Spahr. 2001. Detection and characterization of estrus in dairy cattle with an electronic heatmount detector and an electronic activity tag. J. Dairy Sci. 84:792-798.

Bar, D. 2010. Optimal timing of insemination using activity collars. Pages 100-101 in First North Am. Conf. Precision Dairy Management, Toronto, Canada.

Burke, C. R., S. McDougall, and K. L. MacMillan. 1995. Effects of breed and calving liveweight on postpartum ovarian activity in pasture-fed dairy heifers. Pages $76-78$ in Proc. of the New Zealand Society of Animal Production. D.G. Elvidge, ed. Page Plus, Christchurch, New Zealand.

Cuthbert, S. 2008. DairyNZ milking practices and technology use survey. Page 40 in report prepared for DairyNZ. LIC, Hamilton, New Zealand.

de Mol, R. M., G. H. Kroeze, J. M. F. H. Achten, K. Maatje, and W. Rossing. 1997. Results of a multivariate approach to automated oestrus and mastitis detection. Livest. Prod. Sci. 48:219-227.

Detilleux, J., J. Arendt, F. Lomba, and P. Leroy. 1999. Methods for estimating areas under receiver operating characteristic curves: Illustration with somatic-cell scores in subclinical intramammary infections. Prev. Vet. Med. 41:75-88.

Diskin, M. G., and J. M. Sreenan. 2000. Expression and detection of oestrus in cattle. Reprod. Nutr. Dev. 40:481-491.

Eradus, W. J., W. Rossing, P. H. Hogewerf, and E. Benders. 1992. Signal processing of activity data for oestrus detection in dairy cattle. Pages 360-369 in Proc. Int. Symp. Prospects for Automatic Milking. Pudoc Scientific, Wageningen, the Netherlands.

Fawcett, T. 2006. An introduction to ROC analysis. Pattern Recognit. Lett. 27:861-874.

Harris, B. L., K. Hempstalk, B. Dela Rue, J. Jago, and J. E. McGowan. 2010. Improving the power of activity-based heat detection using additional automatically captured data. Pages 299-302 in Proc. New Zealand Soc. Anim. Prod. R. Sumner, ed. Print House, Hamilton, New Zealand.

Hockey, C. D., J. M. Morton, S. T. Norman, and M. R. McGowan. 2010. Evaluation of a neck mounted 2-hourly activity meter sys- 
tem for detecting cows about to ovulate in two paddock-based Australian dairy herds. Reprod. Domest. Anim. 45:e107-e117.

Lopez, H., L. D. Satter, and M. C. Wiltbank. 2004. Relationship between level of milk production and estrus behavior of lactating dairy cows. Anim. Reprod. Sci. 81:209-223.

MacMillan, K. L., and R. J. Curnow. 1977. Tail painting-A simple form of oestrus detection in New Zealand dairy herds. NZ J. Exp. Agric. 5:357-361.

McDougall, S. 1994. Postpartum anoestrum in the pasture grazed New Zealand dairy cow. PhD Thesis. Massey University, Palmerston North, New Zealand.

McDougall, S., C. R. Burke, N. B. Williamson, and K. L. Macmillan. 1995. The effect of stocking rate and breed on the period of postpartum anoestrum in grazing dairy cattle. Pages 236-238 in Proc. New Zealand Soc. Anim. Prod. D. G. Elvidge, ed. Page Plus, Christchurch, New Zealand.

McGowan, J. E., C. R. Burke, and J. G. Jago. 2007. Validtion of a technology for objectively measuring behaviour in dairy cows and its application for oestrus detection. Pages 136-142 in Proc. New Zealand Soc. Anim. Prod. G. K. Barrell, ed. The Caxton Press, Christchurch, New Zealand.

Newman, M., ed. 2011. DairyNZ Economic Survey 2009-10. DairyNZ Ltd., Newstead, New Zealand.
Roelofs, J. B., F. J. C. M. Van Eerdenburg, N. M. Soede, and B. Kemp. 2005. Pedometer readings for estrus detection and as predictor for time of ovulation in dairy cattle. Theriogenology 64:1690-1703.

Sakaguchi, M., R. Fujiki, K. Yabuuchi, Y. Takahashi, and M. Aoki. 2007. Reliability of estrus detection in Holstein heifers using a radiotelemetric pedometer located on the neck or legs under different rearing conditions. Reprod. Dev. 53:819-828.

Sherlock, R., H. Hogeveen, G. Mein, and M. D. Rasmussen. 2008. Performance evaluation of systems for automated monitoring of udder health: Analytical issues and guidelines. Pages 275-282 in Mastitis Control-From Science to Practice. T. J. G. M. Lam, ed. Wageningen Academic Publishers, Wageningen, the Netherlands.

Verkerk, G. A., R. W. Claycomb, V. K. Taufa, P. Copeman, A. Napper, and E. Kolver. 2001. Cowtrakker ${ }^{\mathrm{TM}}$ technology for improved heat detection. Pages $172-175$ in Proc. New Zealand Soc. Anim. Prod. S. W. Peterson, ed. Design Centre, The Printery, Palmerston North, New Zealand.

Xu, Z. Z., D. J. McKnight, R. Vishwanath, C. J. Pitt, and L. J. Burton. 1998. Estrus detection using radiotelemetry or visual observation and tail painting for dairy cows on pasture. J. Dairy Sci 81:2890-2896. 